\title{
SPECIFIC FEATURES OF IMM TRACKING FILTER DESIGN
}

\author{
Iliyana SIMEONOVA and Tzvetan SEMERDJIEV
}

\section{Introduction}

The Interacting Multiple Model (IMM) estimator is a suboptimal hybrid filter that has been shown to be one of the most cost-effective hybrid state estimation schemes. ${ }^{10}$ The model of hybrid system and the IMM algorithm, initially proposed by Blom, ${ }^{12}$ may serve as a basis for synthesis of more efficient filters for tracking maneuvering aircraft. In this paper we present a number of specific features of the IMM design procedure. In section 2 we briefly describe the hybrid system (aircraft dynamics), hybrid estimation and the principle of the IMM algorithm. The models used in the IMM configuration to describe different flight phases and the measurement model used for our application are presented in sections 3 and 4 respectively. Section 5 provides a detailed discussion on the specific features of the IMM algorithm design. Finally, some examples of IMM tracking filter design and performance evaluation are presented in section 6.

\section{Hybrid Systems and Hybrid Estimation}

\subsection{Hybrid Systems}

An aircraft trajectory can be subdivided into distinct segments, corresponding to modes of flight, ${ }^{10}$ for instance, uniform motion and maneuvers. The multiple model or hybrid system approach assumes the system to be in one of a finite number of modes. ${ }^{2}$ Thus, the aircraft motion can be modeled by a hybrid system that is characterized by two state variables: continuous base - state variable $x(k) \in R^{n_{x}}$ (aircraft position, speed, acceleration, etc.) and a discrete regime variable $j=m_{j}(k) \in M_{r}=1,2 \ldots . r$, which describes the distinct segment of the aircraft trajectory. The transitions (jumps) of the mode variable are modeled with a Markov chain. The nonlinear hybrid system is usually described by the equations:

$$
x(k)=f_{j}[(k-1), x(k-1)]+g_{j}\left[k-1, x(k-1), v_{j}(k-1)\right] \quad \forall j \in M_{r},
$$




$$
z(k-1)=h_{j}[(k-1), x(k-1)]+w_{j}(k-1) \quad \forall j \in M_{r},
$$

where $x(k) \in R^{n_{x}}$ is the system state vector, $z(k-1) \in R^{n_{z}}$ is measurement vector, $v_{j}($.$) and w_{j}($.$) are mode dependent process noise and measurement noise sequences,$ assumed to be white, zero-mean and mutually independent with covariances $Q\left(m_{j}(k)\right)$ and $R\left(m_{j}(k-1)\right)$ respectively. The available measurements for estimation process can be: [range and azimuth]; [range, azimuth and range rate], etc. The $f_{j}[],. g_{j}[],. h_{j}[$.$] are$ known functions. The system at time $(\mathrm{k})$ is assumed to be among $\mathrm{r}$ possible modes, ${ }^{7}$ i.e. $j=m_{j}(k) \in M_{r}=1,2 \ldots . r$, where $j=m_{j}(k)$ denotes that the j-th sub-model is in effect during the sampling period T ending at time (k). $m_{j}(k)_{k=1,2 \ldots}$ is a Markov chain with completely known initial $P_{j}=P\left[m_{j}(0)\right]=j$ and transitional probabilities $p_{i j}=P\left[m_{j}(k) \mid m_{i}(k-1)\right]$.

\subsection{Hybrid Estimation}

The problem of hybrid state estimation is to estimate the base state and the modal state based on noisy measurement sequences. The application of the Multiple - model (MM) method is a major approach to hybrid estimation. It assumes the system obeys one of a finite number of models. The IMM algorithm belongs to the multiple-mode techniques and therefore provides a method to combine the estimates and covariance matrices of each mode with an interacting logic to maintain all of them 'in track. ${ }^{8}$ To estimate the aircraft state, there is a bank of Kalman filters, where each filter matches a model in the set, and a procedure to estimate the probabilities that the target is in each one of the possible modes. Yaakov Bar-Shalom and coauthors provide detailed explanation of the IMM algorithm. ${ }^{1,2,5,10}$ Following Bar-Shalom and Chang, ${ }^{9}$ here we will give a short description of one cycle of the algorithm $(k-1) \rightarrow(k)$ :

\section{Interaction(mixing):}

The mixed initial state $\hat{x}^{0 j}(k-1 \mid k-1)$ and covariance $P^{0 j}(k-1 \mid k-1)$ for the filter matched to mode $m_{j}(k), j=1, \ldots . . r$ are calculated by mixing the state estimates $\hat{x}^{j}(k-1 \mid k-1)$ and covariances $P^{j}(k-1 \mid k-1)$ of all filters obtained at the previous time step

$$
\begin{gathered}
\hat{x}^{0 j}(k-1 \mid k-1)=\sum_{i=1}^{r} \hat{x}^{i}(k-1 \mid k-1) \mu_{i \mid j}(k-1 \mid k-1) \\
P^{0 j}(k-1 \mid k-1)=\sum_{i=1}^{r} \mu_{i \mid j}(k-1 \mid k-1) P^{i}(k-1 \mid k-1)+ \\
{\left[\hat{x}^{i}(k-1 \mid k-1)-\hat{x}^{0 j}(k-1 \mid k-1)\right]\left[\hat{x}^{i}(k-1 \mid k-1)-\hat{x}^{0 j}(k-1 \mid k-1)\right]^{T},(4)}
\end{gathered}
$$


where

$$
\mu_{i \mid j}(k-1 \mid k-1)=\left(1 / \overline{c_{j}}\right) p_{i j} \mu_{i}(k-1)
$$

are mixing probabilities, and $\overline{c_{j}}=\sum_{i=1}^{r} p_{i j} \mu_{i \mid j}(k-1 \mid k-1)$ is the normalization factor.

\section{Mode-conditioned filtering:}

The estimate (3) and covariance (4) are used as input to the Kalman filter (linear or extended) matched to $m_{j}(k)$ to obtain state estimate $\hat{x}^{j}(k \mid k)$ and covariance $P^{j}(k \mid k)$ at time $\mathrm{k}$, as well as the mode likelihood $\Lambda_{j}(k)$ :

$$
\Lambda_{j}(k)=\left|S_{j}(k)\right|^{-1 / 2} \exp \left\{-\frac{1}{2} \tilde{z}_{j}^{T}(k) S_{j}^{-1}(k) \tilde{z}_{j}(k)\right\},
$$

where $\tilde{z}_{j}(k)$ and $S_{j}(k)$ are innovation and its covariance of the $j$ conditional filter.

\section{Mode probability update:}

The model probabilities $\mu_{j}(k)$ are updated as follows:

$$
\mu_{j}(k)=\frac{1}{c} \Lambda_{j}(k) \sum_{i=1}^{r} p_{i j} \mu_{i}(k-1)=\frac{1}{c} \Lambda_{j}(k) \overline{c_{j}}, \quad c=\sum_{j=1}^{r} \Lambda_{j}(k) \overline{c_{j}},
$$

where $c$ is the normalization factor.

\section{Estimate and covariance combination}

The combination of the updated mode conditioned estimates and covariances produces the output estimates:

$$
\begin{gathered}
\bar{x}(k \mid k)=\sum_{j=1}^{r} \hat{x}^{j}(k \mid k) \mu_{j}(k), \\
\left.P(k \mid k)=\sum_{j=1}^{r} P_{j}(k \mid k)+\left[x_{j}(\bar{k} \mid k)-\bar{x}(k \mid k)\right]+x_{j}(\bar{k} \mid k)-x(\bar{k} \mid k)^{T}\right] \mu_{j}(k) .
\end{gathered}
$$

The IMM algorithm has three desirable properties: it is recursive, modular and has fixed computational requirements per cycle. The IMM algorithm can use as its building blocks Kalman filters (KF) or Extended Kalman filters (EKF) to account for nonlinearities in the measurement equation (for range - azimuth - elevation observations), and in the state equation (for coordinated turns) or probabilistic data association filters based on KF or EKF when data association is a major problem.

\section{Aircraft Motion Models}

Civilian aircraft in air traffic control (ATC) systems have two basic modes of flight: 
- uniform motion (UM) - the straight and level flight with a constant speed and heading and

- Maneuver - turning or climbing/descending. ${ }^{1}$

Let us consider the linear version of equation(1) to aircraft trajectory modeling in two Cartesian coordinates (x, y):

$$
X(k)=F_{j} \cdot X(k-1)+G_{j} \cdot v_{j}(k-1),
$$

where $v_{j}(k-1)=\left[\begin{array}{ll}v_{j x}(k-1) & v_{j y}(k-1)\end{array}\right]^{T}$ are white noise sequences used to model uncertain accelerations. The model extension in $(\mathrm{x}, \mathrm{y}, \mathrm{z})$ coordinates is straightforward. In this section we will present some of the most commonly used aircraft motion models.

Notations: $X \equiv X(k) ; F_{j} / G_{j}$ - transition/noise gain matrix for both coordinates (x, $\mathrm{y}) ; f_{j} / g_{j}$ - transition/noise gain matrix for each coordinate $\mathrm{x} / \mathrm{y}$.

\subsection{Piecewise Constant White Acceleration Model}

This is a second order (nearly constant velocity) model with the following parameters:

- State space vector: $X=\left[\begin{array}{llll}x & \dot{\mathrm{x}} & y & \dot{\mathrm{y}}\end{array}\right]^{T}$;

- Transition matrix: $F_{1}=\operatorname{diag}\left[f_{1}, f_{1}\right]$, where $f_{1}=\left[\begin{array}{ll}1 & T \\ 0 & 1\end{array}\right]$;

- Noise gain: $G_{1}=\operatorname{diag}\left[g_{1}, g_{1}\right]$, where $g_{1}=\left[\begin{array}{ll}T * T / 2 & T\end{array}\right]^{T}$.

This model assumes the variations in velocity components for each coordinate are piesewise constant zero-mean white noise accelerations. ${ }^{1}$ The process noise variances in each coordinate are assumed to be equal: $\sigma_{\nu x}^{2}=\sigma_{\nu y}^{2}=q$ and $\sigma_{\nu x \nu y}=0$.

- A 'nearly constant velocity motion model' $\left(M_{1}\right)$ for the UM modeling is obtained by the choice of 'small' noise values: $q=q_{1} \cdot 1,6$

- The same model, but with higher levels of process noise $q=q_{2}$ can model 'rough' maneuvers. This model is denoted as $M_{2}$.

\subsection{Piecewise Constant Wiener Process Acceleration Model}

This is a third order (nearly constant acceleration) model used to describe the maneuvering phase of flight. It has the following parameters:
- State space vector: $X=[x$
$\ddot{x}$
y $\quad \ddot{y}]^{T}$; 
- Transition matrix: $F_{3}=\operatorname{diag}\left[f_{3}, f_{3}\right]$, where $f_{3}=\left[\begin{array}{ccc}1 & T & T * T / 2 \\ 0 & 1 & T \\ 0 & 0 & 1\end{array}\right]$;

- Noise gain: $G_{3}=\operatorname{diag}\left[g_{3}, g_{3}\right]$, where $g_{3}=\left[\begin{array}{ll}g_{1} & 1\end{array}\right]^{T}$.

This model assumes the acceleration increments for each component during $\mathrm{k}$-th sampling period are zero-mean white sequence. ${ }^{2}$

- A 'nearly constant acceleration motion model' $M_{3}$ is obtained by the choice of 'small' $q=q_{3} \cdot{ }^{1,6}$

- The model $M_{4}$ is the same but with higher levels of process noise $q=q_{4} \cdot{ }^{6}$

\subsection{Coordinated Turn Models (CTM)}

Coordinated turn models are another way to describe the maneuvering mode of flight. The turning of civilian aircraft usually follows a pattern known as a "coordinated turn": that means the target is moving with constant speed and turning with constant turn rate. There are two basic coordinated turn models:

Constant turn rate models: here turn rate $\omega$ is a completely known design parameter. The models $M_{5}$ and $M_{6}$ are used for a left-hand turn $(\omega>0)$ and for a right-hand turn $(\omega<0)$, respectively. This assumption is suitable for a civilian aircraft because its maneuvers are constrained by flight rules, especially when approaching an airport. ${ }^{5}$ The state space vector and noise gain coincide with those of models $M_{1}$ and $M_{2}$, but the transition matrix is different:

- Transition matrix :

$$
F_{5,6}=\left[\begin{array}{l}
1 \\
0 \\
0 \\
0
\end{array}\right.
$$

$$
\begin{array}{cc}
\omega^{-1} \sin (\omega \cdot T) & 0 \\
\cos (\omega \cdot T) & 0 \\
\omega^{-1}(\omega-\cos (\omega \cdot T)) & 1 \\
\sin (\omega \cdot T) & 0
\end{array}
$$

$$
\left.\begin{array}{c}
\omega^{-1}(\omega-\cos (\omega \cdot T)) \\
-\sin (\omega \cdot T) \\
\omega^{-1} \sin (\omega \cdot T) \\
\cos (\omega \cdot T)
\end{array}\right]
$$

- The process noise variances in each coordinate are $q_{5}$ and $q_{6}$ respectively.

For military aircraft the above assumption is less natural, so the model $M_{7}$ presents the case where $\omega$ is not known. What we need here is to augment the state space vector with unknown turn rate $\omega$.

- The discrete time state space equation is nonlinear, because the transition matrix is a function of the state component $(\omega)$ :

$$
X(k)=F_{7}(\omega) \cdot X(k-1)+G_{7} \cdot v_{7}(k-1) .
$$


- State space vector: $X=\left[\begin{array}{lllll}x & \dot{\mathrm{x}} & y & \dot{\mathrm{y}} & \omega\end{array}\right]^{T}$;

- Transition matrix: $F_{7}=\left[\begin{array}{c|c}F_{5,6} & 0 \\ \hline 0 & 1\end{array}\right]$;

- Noise gain: $G_{7}=\operatorname{diag}\left[g_{7}, g_{7}\right]$ where $g_{7}=\left[g_{1}\right.$

Here $v_{7}(k-1)=\left[\begin{array}{ll}v_{7 x}(k-1) & v_{7 y}(k-1)\end{array}\right]^{T}$ are white noise sequences used to model uncertain accelerations in $\mathrm{x}$ and y coordinates due to uncertainty in $\omega$.

- The process noise variance is $q_{7}$.

In all the above models $\sigma_{\nu_{x} \nu_{y}}=0$. Furthermore, there are two basic principles to maneuver modeling ${ }^{5}$ : exact maneuver modeling and approximate maneuver modeling. Thus, models $M_{2}, M_{3}, M_{4}$ are based on approximate modeling, while models $M_{5}, M_{6}, M_{7}$ assume exact modeling. The reader is referred to the works of BarShalom, $\mathrm{Li}$ and coauthors ${ }^{1,2,5,6}$ for a comprehensive presentation of aircraft motion modeling.

\section{Measurement Model}

Let us consider the case when measurement (sensor modeling) equation (2) is not mode dependent:

$$
z(k-1)=h[(k-1), X(k-1)]+w(k-1) .
$$

For our application the nonlinear measurement function $h[$.] of (12) converts Cartesian target state space vector coordinates $(\mathrm{x}, \mathrm{y})$ to $\mathrm{z}=[\text { range and azimuth }]^{T}, h[]=.\left[h_{1} h_{2}\right]^{T}$, where $h_{1}=\sqrt{x^{2}+y^{2}}$ and $h^{2}=\arctan \frac{x}{y}$.

Due to the nonlinearity of the measurement(sensor) equation (12), the Extended Kalman filter is used in the IMM configuration. It requires linearization of $h[$.$] about the posi-$ tion prediction, that yields Yacobian.

\section{Specifics of the IMM Tracking Filter Design}

In this section we shall provide the practitioners with some practical rules how to chose the best IMM filter design parameters.

To obtain the best possible results, the IMM algorithm has to be properly designed. The designer need to take into account ${ }^{1}$ :

- Accuracy in estimating both position and velocity. A trade-off between the peak errors and the errors during uniform motion is desirable;

- Timeliness of the maneuver detection and termination;

- Complexity of the implementation. 
The design of an IMM estimator consist of the following steps ${ }^{1}$ :

- Selection of the set of models describing aircraft dynamics and their structure;

- Selection of the process noise intensities for the various models;

- Selection of the Markov chain transition probabilities.

\subsection{Model Set Selection}

Designing IMM tracking filters, both complexity and quality of the models need to be considered. Typically, the models used in the IMM configuration include one nearly constant velocity motion model for non-maneuvering regime of flight and a set of exact or approximate maneuver models for the maneuvering phases. The models for uniform and maneuvering motion are presented in the previous section. Additionally, we need to account that increasing the number of conditional sub-models to cover the uncertain behavior of highly maneuvering objects increases considerably the computation load but does not guarantee better performance due to the competition among models. ${ }^{3}$ The precise modeling of every aircraft trajectory segment will lead to more accurate results especially in speed estimation. ${ }^{5}$

\subsection{Process Noise Selection}

The selection of process noise standard deviations for each model is an important part of the estimator design. The process noise levels are selected based on the expected disturbances and target maneuvering magnitudes. ${ }^{1}$ Let us consider some of the models presented in the previous section.

- The small process noise of model $M_{1}$ accounts for air turbulence, winds aloft changes, ${ }^{2}$ slow turns, as well as small linear accelerations.

- The high level process noise of model $M_{2}$ allows for target acceleration and is applicable (with limited degree of success) to tracking maneuvering target. ${ }^{2}$ The process noise range is usually selected as follows: 0.5. $a_{\max } \leq q_{2} \leq a_{\max }{ }^{2}$ where $a_{\max }$ is the maximum acceleration magnitude. According to Bar-Shalom and $\mathrm{Li},{ }^{2}$ the IMM configuration with $M_{1}$ and $M_{2}$ models does obtain acceptable results for maneuvers with turn rates up to $3 \mathrm{deg} / \mathrm{s}$; it does not, however, yield good estimates for faster turns.

- The $M_{3}$ model with low process noise provides more accurate estimation during a maneuver.

- A maneuver onset is a rapid jump to a non-zero target acceleration from zero and then a jump back to zero acceleration at termination. ${ }^{1}$ So, the $M_{4}$ model with high level process noise can model more precisely maneuver onset and termination. The noise range should be of the order of the magnitude of the maximum 
acceleration increment over a sampling period: $0.5 \triangle a_{\max } \leq q_{4} \leq \triangle a_{\max }{ }^{2}$ The IMM configuration with one second order model and two third order models with different noise levels ${ }^{3,5,7}$ is best suitable for estimating more intensive ( $a_{n}=7 \mathrm{~g}$ ) maneuvers with short duration, as well as longitudinal acceleration. But it leads to considerable errors for moderate turns of $1-5 \mathrm{~g}$. Additionally, the peak errors are not significantly reduced compared to those obtainable by using the single-model filter. ${ }^{5}$ The explanation is that the three models are not discriminating enough. Also, the interaction step mixes the regime-conditioned estimates in a way that helps the filters based on the "wrong" models to come back on track. In this case, the a posteriori information conveyed by the innovations conditioned on the mode hypothesis do not have enough contrast. According to Kirubarajan and coauthors, ${ }^{11}$ an IMM configuration which uses as it building modules models $M_{1}, M_{2}$ and $M_{4}$ is most suitable for highly maneuvering targets. Finally, the use of the maneuver detection model $M_{4}$ is not necessary when tracking civilian aircraft.

- The right choice of the noise levels $q_{5,6}$ of models $M_{5}$ and $M_{6}$ depends on the expected turn rate and on the number of models to be used in estimating maneuvers. The standard deviation of the process noise can be selected as $q_{5,6}=0.5 .\left(\omega_{i+1}-\omega_{i}\right) . V=0.5 . \triangle U,{ }^{4}$ where $\omega_{i+1}, \omega_{i}$ are turn rates of two adjacent models and $V$ is the expected linear speed . The IMM configuration could include one uniform motion model and a set of different constant turn rate models. According to Munir and Atheron, ${ }^{4}$ the use of coordinated turn models with known $\omega$ is better than IMM with estimated $\omega$ when the models in the former case fully "cover" the turn rate of target motion and vice-versa.

- Because of the delay in estimating $\omega$ at the onset of the maneuver, the use of model $M_{7}$ produces rather large peaks. However, once the $\omega$ estimate converges, a very good tracking performance is obtained during turns. ${ }^{5}$ According to an earlier work of the authors, ${ }^{3}$ using model $M_{7}$ is best suitable for tracking aircraft performing maneuvers with moderate, a priori unknown normal acceleration $\left(a_{n}=1 \div 5 \mathrm{~g}\right)$, as well as for more complex maneuvers with longitudinal and transversal accelerations.

The practitioner should also be aware that a high degree of smoothing and, thus, a low value of the convergence noise at uniform motion leads to high peaks during maneuvers, and vice versa. ${ }^{8}$

\subsection{Transition Probabilities}

The Markov chain transition probabilities are related to the expected sojourn time in the various modes. ${ }^{1}$ These probabilities are chosen according to the designer's beliefs 
about the frequency of change from one mode to the rest. They can be subsequently adjusted by means of Monte Carlo simulations. The guideline for a proper choice is to match roughly the transition probabilities with the actual mean sojourn time $\left(\tau_{i}\right)$ of each mode, ${ }^{2}$ the diagonal coefficients being determined as $p_{i i}=1-\frac{1}{E\left[\tau_{i}\right]}$. The transition probabilities $p_{i j}$ for $i \neq j$ are selected using the identity: $\sum_{j \neq i} p_{i j}=1-p_{i i}$.

The choice of the transition probabilities provides a certain degree of trade-off between the peak estimation errors at the onset of the maneuver and the maximum reduction of the estimation errors during the uniform motion. ${ }^{2}$ Following Bar-Shallm and Li, Herrero and coauthors, ${ }^{2,8}$ the practitioner should be aware that high transition probabilities lead to low peak errors during maneuver, but at the cost of low smoothing and higher errors when tracking uniform motion. Also, the transitions between models are quickly detected and the filter is very adaptive. Let us consider an IMM configuration with a $M_{1}$ model and a model with unknown $\omega-M_{7}$. According to Bar-Shalom, ${ }^{5}$ if the transition matrix of this configuration has large off-diagonal entries, then it favors regime transition and results in much more volatile sample paths of the probabilities. This phenomenon is called "regime mixing." Here, the peak errors are so low that the occurrence of the maneuver is hardly noticeable.

\subsection{Initial Probabilities}

If initial probabilities are set to be equal in orded to account for the worst case of uncertainty, then the initial estimation errors will be large. ${ }^{2}$

\section{Performance Analysis}

\subsection{Target Motion Scenario}

In order to test the capabilities of different IMM configurations we consider a class of maneuvering aircraft performing sweep maneuvers with normal acceleration up to $7 \mathrm{~g}$ $\left(\mathrm{g} \approx 9.8 \mathrm{~m} / \mathrm{s}^{2}\right)$. The scenario of motion is depicted on Figure 1.

\subsection{Sensor Parameters}

The simulation involves a single track while scan (TWS) radar with scanning period of $1 \mathrm{~s}$. The sensor parameters considered here are : range and azimuth accuracy: $\sigma_{\mathrm{D}}=$ $120 \mathrm{~m}$ and $\sigma_{\beta}=0.2 \mathrm{deg}$, respectively.

\subsection{IMM Tracking Filter Design}

Here we introduce three different sets of models, respectively IMM2, IMM3, IMM$\mathrm{CT}$, to describe the target motion scenario. Their design parameters are: 
- IMM2 - mode set: $\left[M_{1}, M_{3 I}\right]$; -process noises: $\sigma_{1}=2.5 \mathrm{~m} / \mathrm{s}^{2}, \sigma_{3 I}=20.5 \mathrm{~m} / \mathrm{s}^{2}-$ I - intermediate noise; -transition probabilities $p_{11}=0.9, p_{21}=0.20$.

- IMM3 - mode set: $\left[M_{1}, M_{3}, M_{4}\right]$; -process noises: $\sigma_{1}=2.5 \mathrm{~m} / \mathrm{s}^{2}, \sigma_{3}=7 \mathrm{~m} / \mathrm{s}^{2}$, $\sigma_{4}=40 \mathrm{~m} / \mathrm{s}^{2} ;$-transition probabilities $p_{11}=0.9, p_{12}=0.05, p_{21}=0.15$, $p_{22}=0.75, p_{31}=0.20, p_{32}=0.05$.

- IMM-CT -mode set: $\left[M_{1}, M_{5}, M_{6}\right]$; -process noises: $\sigma_{1}=2.5 \mathrm{~m} / \mathrm{s}^{2}$, $\sigma_{5,6}=3.5 \mathrm{~m} / \mathrm{s}^{2}, \omega_{5,6}= \pm 0.233 \mathrm{rad} / \mathrm{s} ;$-transition probabilities $p_{11}=0.9$, $p_{12}=0.05, p_{21}=0.20, p_{22}=0.80, p_{31}=0.20, p_{32}=0.00$.

\subsection{Performance Evaluation and Analysis}

The IMM filters' efficiency was evaluated according to the root mean-square(RMS) error both in position and in velocity. The results presented here are based on $\mathrm{Nr}=100$ Monte Carlo runs. RMS is defined as

$\sigma_{\mathrm{pos}}=\sqrt{\frac{1}{N} \cdot \sum_{i=1}^{N}\left[x^{i}(k)-\hat{x}^{i}(k \mid k)\right]^{2}+\left[y^{i}(k)-\hat{y}^{i}(k \mid k)\right]^{2}}$, where the superscript $\mathrm{i}$ denotes the results from run $\mathrm{i}$, and $\mathrm{x}(\mathrm{k}), \mathrm{y}(\mathrm{k})$ are true target positions $(\mathrm{x}, \mathrm{y})$. The equation for RMS error in estimating velocity is derived likewise.

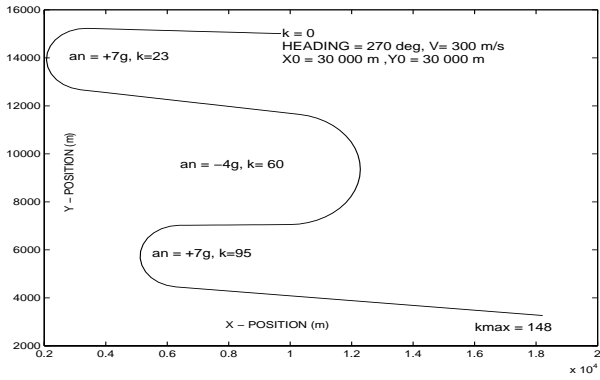

Figure 1: Target Trajectory.

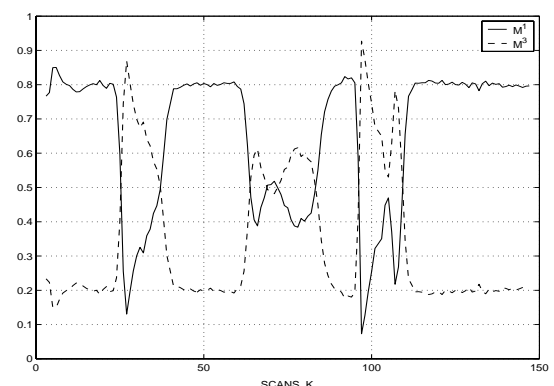

Figure 2: IMM2. Mode probabilities.

The analysis of simulation results is summarized as follows:

- The average mode probabilities for each case over scans are depicted on Figures 2, 3 and 4. The correct mode has the largest probability during each segment. ${ }^{5}$

- Comparison of RMS position and velocity errors are shown on Figures 5 and 6 , respectively. The general behavior of these error curves is typical for IMM algorithms. Natural transients are observed at the onset and termination of maneuvers. The peaks at the start and the end of a maneuver are caused by the delay of mode probabilities switching from one mode to another. After switching, the slower decrease of the errors corresponds to the convergence of maneuver filter. ${ }^{5}$ 


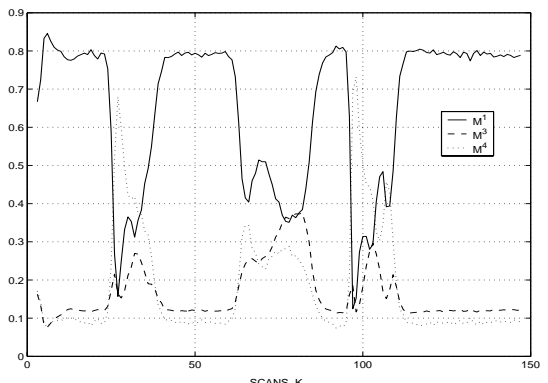

Figure 3: IMM3. Mode probabilities.

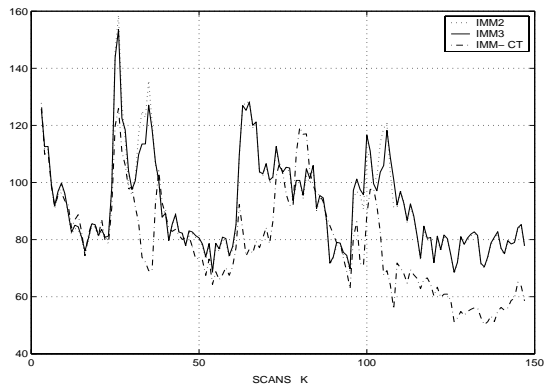

Figure 5: RMS position errors [m]

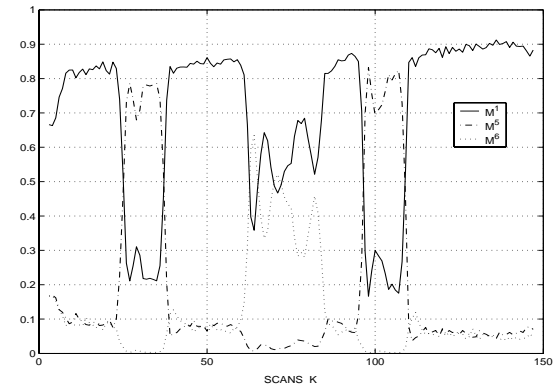

Figure 4: IMM-CT. Mode probabilities

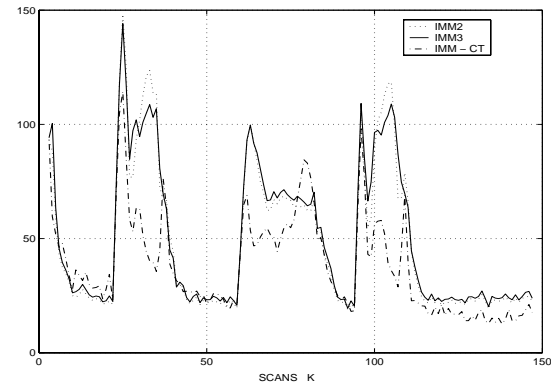

Figure 6: RMS velocity errors $[\mathrm{m} / \mathrm{s}]$

As expected, the use of model $M_{4}$ in IMM3 reduce the peak errors both in position and in velocity. Also, the use of exact maneuver models as in IMM-CT reduces significantly the peak errors and the errors during uniform motion.

\section{Conclusions}

Considerable number of publications is devoted to the design of IMM tracking filters. In this article we summarize results, conclusions and experience of various authors in order to provide researchers and designers with a fast and easy way to determine the advantages and the capacity of different IMM structures in variety of target motion scenarios. This is an objective not addressed in available bibliography on IMM tracking filters. The simulation results are obtained using the comprehensive MATLAB tool developed by the team at the Central Laboratory for Parallel Processing of the Bulgarian Academy of Sciences. This tool could be used as a basis for a synthesis of many other motion scenarios and IMM tracking filters. 


\section{Acknowledgement}

This research work was partially supported by Center of Excellence BIS21 grant ICA12000-70016.

\section{Notes}

1 Yaakov Bar-Shalom, Xiao-Rong Li, Multitarget-multisensor tracking: principles and techniques (Storrs, CT: YBS Publishing, 1995).

2 Yakov Bar-Shalom, Xiao-Rong Li, Estimation and tracking: principles,techniques and software (Boston, MA: Artech House, 1993).

3 Donka Angelova, Vesselin Jilkov, Tzvetan Semerdjiev, "State estimation of a nonlinear dynamic system by parallel algorithm," in Proc. 2nd EUropean Nonlinear Oscilation Conference EUROMECH (Prague, 9-13 September 1996), 215-218.

4 A. Munir, D. Atheron, "Maneuvering target tracking using different turn rate models in the interacting multiple model algorithm," in Proc. of the 34th Conf. on Decision and Control (New Orleans, LA: Desember 1995), 2747-2751.

5 Yaakov Bar-Shalom, Multitarget-Multisensor Tracking: Aplications and Advances, Volume II, (Boston, MA: Artech House, 1993).

6 D. Lero, Yaakov Bar-Shalom, "Interactive Multiple Model Tracking with Target Amplitude Features," IEEE Trans. Aerospace and electronic systems AES-29 (1993): 495-508.

7 Donka Angelova,Vesselin Jilkov, Tzvetan Semerdjiev, "Tracking Maneuvering Target by Interacting Multiple model," Comtes rendus de l'Academie bulgare des Sciences 49, (1996): 37-40.

8 J. Herrero, J. Portas, J. López, Vela, J. Garcia, "Interactive Multiple Model Filter Optimisation Tool for Air Traffic Control Applications," in Proc. Fourth Annual Conference on Information Fusion, (Montréal, Québec, Canada, 2001), TuB2-19-TuB2-26.

9 Yaakov Bar-Shalom, K.C. Chang, "Tracking a Maneuvering Target Using Input Estimation Versus the Interacting Multiple Model Algorithm," IEEE Trans. Aerospace and electronic systems AES-25 (1989): 296-300.

10 E. Mazor, A. Averbuch, Yakov Bar-Shalom, J. Dayan, "Interacting Multiple Model Methods in Target Tracking: A Survey," IEEE Trans. Aerospace and electronic systems AES34 (1998): 103-123.

11 T. Kirubarajan, Yaakov Bar-Shalom, W. Blair, G. Watson, "IMMPDA Solution ot Benchmark for Radar Resource Allocation and Tracking in the Presence of ECM," IEEE Transactions on Aerospace and electronic systems 30, 2 (August 1996).

12 Helk A.P. Bloom, "An Efficient Decision-Making-Free Filter for Processes with Abrupt Changes," IFAC Symp. on Identification and Systhem Parameter Estimation, (York, United Kingdom, July 1985).

ILIYANA SVETLOMIROVA SIMEONOVA is a Ph.D. student at the Central Laboratory for Parallel Processing, Bulgarian Academy of Sciences. She received M.Sc. degree from the Technical University of Sofia in 2000. E-mail: iliyana@bas.bg.

TZVETAN ATANASOV SEMERDJIEV - see p. 90. 